\title{
EL EFECTO DE LA DESHIDRATACION EN EL RENDIMIENTO ANAEROBICO
}

\author{
Fernando Ubiratan Da Silveira \\ Escuela de Educación Física y Deportes \\ Universidad de Costa Rica, San José, Costa Rica \\ E-mail: judobr@yahoo.com.br
}

\begin{abstract}
Resumen
Da Silveira, F. U. (2006). El Efecto de la Deshidratación en el Rendimiento Anaeróbico. Revista de Ciencias del Ejercicio y la Salud, 4(1), 13-21. El aumento de la temperatura corporal y la aparición de la sudoración son dos respuestas normales a la actividad física. Sin embargo, la deshidratación y la hipertermia que frecuentemente acompañan el entrenamiento deportivo y a la competición, son las causas más comunes y al mismo tiempo evitables de la fatiga prematura entre los atletas (Murray, 1996). La deshidratación contribuye a la hipertermia reduciendo la capacidad del organismo de perder calor, y aún bajos niveles de deshidratación de tan sólo un 2\% del peso corporal perdido, pueden perjudicar el rendimiento físico (Maughan, 2003; Murray, 1996). Los efectos de la deshidratación sobre variables anaeróbicas no son muy claros, debido a los diferentes porcentuales y métodos de deshidratación utilizados en las investigaciones. Por eso, la presente revisión de literatura propone un análisis de los efectos de la deshidratación sobre el rendimiento y metabolismo anaeróbico bajo la organización según tres distintos modelos experimentales: 1. métodos de deshidratación pasiva; 2. métodos de deshidratación activa; 3. método de deshidratación mixto. Así como ocurre en el rendimiento aeróbico, las diferencias en los resultados del rendimiento anaeróbico parecen estar relacionados con algunos factores como: la magnitud de cambio en el peso corporal, el tiempo en que los sujetos estuvieran bajo proceso de deshidratación, las pruebas utilizadas para medir las variables anaeróbicas, los métodos de deshidratación y principalmente si hubo combinación de estos métodos. PALABRAS CLAVES: Deshidratación, Deporte, Rendimiento, Anaeróbico.
\end{abstract}

\section{INTRODUCCIÓN}

El agua es la sustancia más abundante en
el cuerpo humano, compone aproximadamente entre un $50-60 \%$ del peso total del cuerpo y tiene gran importancia en el mantenimiento de la vida (Maughan, 2003).

Los cambios agudos en el peso corporal durante el ejercicio normalmente ocurren, principalmente, por la pérdida de agua en forma de sudor. Esas pérdidas deben ser lo más prontamente restituidas para que no resulten en efectos negativos en el rendimiento físico, tampoco en la salud (Shirreffs, 2000). Desde por lo menos cinco décadas de investigaciones en el área de la deshidratación, se ha demostrado que la deshidratación durante el ejercicio altera las funciones de los sistemas cardiovascular, termorregulador, metabólico y endocrino. El deterioro de estos sistemas debido a la deshidratación puede acelerar la aparición de la fatiga en ejercicios aeróbicos de larga duración (González-Alonso y Coyle, 1998). Entretanto, los efectos de la deshidratación en el rendimiento anaeróbico no están completamente claros. En esta revisión de literatura se presentan los mecanismos fisiológicos responsables del balance de líquidos, los mecanismos de termorregulación, diferentes métodos de deshidratación, así como sus efectos en el rendimiento y metabolismo anaeróbico.

\section{El balance de líquidos:}

Las pérdidas de agua corporal se dan a través de la piel -sudor-, por el tracto 
respiratorio -vapor de agua en el aire espirado-, por el tracto gastrointestinal heces- y los riñones -orina (Wilmore y Costill, 1999).

La pérdida diaria de agua para un individuo sedentario saludable es de aproximadamente 2,6 litros, esas pérdidas se dan a través de excreción de la orina (1,5 1), por el tracto respiratorio $(0,41)$, por las heces $(0,21)$ y por sudoración $(0,51)$. Entonces, la cantidad de agua para el mantenimiento de las funciones corporales es de cerca de 2,6 litros diarios para sedentarios, que proviene de la ingesta de líquidos (1,3 1), del agua de los alimentos ( 11 1) y del metabolismo celular $(0,3$ 1) (Shirreffs, 2000; Wilmore y Costill, 1999). El agua metabólica se produce cuando se degradan los nutrientes y representan cerca de $25 \%$ de los requerimientos diarios de líquido (McArdle, Katch y Katch, 1986) la oxidación completa de $100 \mathrm{~g}$ de carbohidratos, proteínas y grasas produce 55, 41 y $107 \mathrm{~g}$ de agua.

Según Koppen y Stanton (2001), la ADH (hormona anti-diurética) actúa en los riñones regulando el volumen y la osmolaridad de la orina; cuando disminuye la $\mathrm{ADH}$ en la sangre, aumenta la diuresis, cuando aumenta la $\mathrm{ADH}$, disminuye la diuresis.

La aldosterona es la hormona esteroide cuya principal función es aumentar la reabsorción de sodio en el túbulo renal, ocasionando un aumento de secreción de potasio y junto con la $\mathrm{ADH}$, aumenta la reabsorción de agua en la nefrona. La liberación de aldosterona está influenciada por muchos factores. Uno de esos factores es el sistema renina-angiotensina, el cual consiste en que cuando ocurre una disminución del volumen sanguíneo, se da la producción de renina por parte de los riñones, y por medio de la angiotensina II se produce un aumento en la secreción de aldosterona (Shirreffs y Maughan, 2001).

La cantidad de líquido que un individuo pierde puede ser estimado con un alto grado de confiabilidad si se miden los cambios en el peso corporal durante el período de tiempo en el que se da esta pérdida. Sin embargo, es más difícil estimar las pérdidas de electrolitos, ya que la composición del sudor es más difícil de medir, además de que hay grandes variaciones entre individuos, y en el mismo individuo, la composición del sudor varía en el tiempo. Los principales electrolitos que se pierden en el sudor son el sodio y el cloro (Aragón-Vargas, Maughan, Rivera-Brown, Meyer, Murray, Barros, García, Sarmiento, Arroyo, Javornik, Matsudo, Salazar y Lentini, 1999; Maughan, 1998).

En condiciones de reposo, el contenido de agua del cuerpo permanece relativamente estable y la pérdida de fluidos es equivalente al consumo de éstos (Wilmore y Costill, 1999). Sin embargo, cuando se realiza ejercicio en condiciones ambientales extremas, las tasas de sudoración pueden exceder los 2 litros por hora (Maughan, 1998).

Un consumo inadecuado de líquidos durante el ejercicio, afectará la regulación de la temperatura corporal, la función cardiovascular y el metabolismo del músculo (Horswill, 1998). No obstante, el efecto negativo de la deshidratación en el rendimiento deportivo y en la salud puede verse atenuado por el consumo de líquidos para compensar las pérdidas por sudoración (Maughan, 2003).

\section{Los mecanismos de Regulación de la Temperatura Corporal}

La temperatura corporal refleja la existencia de un cuidadoso equilibrio entre la producción y pérdida de calor. Siempre que este equilibrio se altera, la temperatura de nuestro cuerpo cambia. Normalmente estas fluctuaciones no suelen ser superiores a $1,0^{\circ} \mathrm{C}$. Sin embargo durante la realización de ejercicios intensos prolongados, enfermedades o condiciones ambientales extremas de calor y de frío, las temperaturas corporales se desvían fuera de su rango normal de variación, que está entre 36,1 y 37, $8^{\circ} \mathrm{C}$ (Maughan, 2003; Wilmore y Costill, 1999). 
Los tejidos metabólicamente activos producen calor que puede usarse para mantener la temperatura interna corporal. La capacidad para mantener una temperatura interna constante depende de la capacidad para equilibrar el calor que se obtiene del metabolismo y del ambiente, con el calor que pierde nuestro cuerpo (Ruiz, Mesa, Mula, Gutiérrez y Castillo, 2003).

La producción de calor corporal es directamente proporcional a la intensidad y duración del ejercicio que se realiza (AragónVargas et al, 1999). Según Wilmore y Costill (1999), para que el cuerpo pueda perder calor al ambiente, es necesario que este calor llegue al exterior. El calor de las partes internas del cuerpo es transportado por la sangre hacia la piel donde puede ser transferido al ambiente por conducción, convención, radiación o evaporación. La evaporación es el camino más importante para la disipación del calor durante el ejercicio. Cuando la temperatura corporal se eleva, la producción de sudor aumenta, haciendo que se humedezca la piel y la evaporación de esta humedad disminuye el calor de la superficie de la piel. Este medio de transferencia de calor representa cerca de $80 \%$ de la pérdida total de calor durante actividades físicas. Por lo tanto, la evaporación del sudor adquiere una importancia creciente a medida que la temperatura corporal aumenta.

Los cambios en la temperatura corporal son detectados por dos grupos de termorreceptores:

a) Los receptores centrales, que están localizados en el hipotálamo y detectan la temperatura de la sangre cuando ésta circula a través del cerebro, de modo que al haber cambios en la temperatura de la sangre que pasa a través del hipotálamo, se activan reflejos que ayudan a conservar o eliminar el calor corporal según las necesidades.

b) Los receptores periféricos, localizados en la piel, encartados de detectar la temperatura ambiental y de enviar la información al hipotálamo y a la corteza cerebral, permitiendo percibir concientemente la temperatura del ambiente (Wilmore y Costill, 1999).

\section{La deshidratación y el rendimiento deportivo:}

Para que el atleta logre su mejor resultado, o sea, que rinda el máximo en su deporte, implica una infinita cantidad de variables interrelacionadas. No es solamente, la condición fisiológica óptima, sino también la psicológica y motriz (Franchini, 2001).

Durante competiciones o prácticas deportivas intensas se suelen producir cambios significativos en el peso corporal, provocados principalmente, por la pérdida de agua en forma de sudor (Shirreffs, 2000). Esto puede alterar la homeostasis del volumen intra y extracelular del organismo y producir alteraciones importantes en las funciones corporales implicándose, entre otros, los sistemas nervioso, cardiovascular, termorregulador, metabólico, endocrino o excretor. Todos ellos pueden perjudicar las capacidades físicas y psicológicas durante el ejercicio (González-Alonso y Coyle, 1998; Solera, 2003; Leiberman, Bathalon, Falco, Kramer, Morgan y Niro, 2005; Maughan, 2003).

Cuando el organismo tiene niveles adecuados de hidratación, se dice que presenta un estado de euhidratación. La deshidratación es definida como la pérdida de agua corporal debido al sudor a lo largo de un ejercicio físico sin reposición de fluidos, o donde la reposición de fluidos no compensa la proporción de fluido perdido. En contraste, la hipohidratación se refiere al estado de hidratación tras la pérdida de una cierta cantidad de agua corporal. La deshidratación puede producirse por un aumento de las pérdidas hídricas, por un menor aporte de líquidos o por la combinación de ambos factores (Cheung, Mclellan y Tenaglia, 2000; Lamb y Shehata, 1999).

De acuerdo con Aragón-Vargas et al, (1999); Lieberman et al, (2005); Maughan, (2003); y Murray, (1996), algunos de los 
efectos fisiológicos de la deshidratación son: disminución del volumen sanguíneo, aumento de la osmolaridad de la sangre, aumento de la frecuencia cardiaca como mecanismo para mantener el gasto cardiaco, disminución del flujo sanguíneo a la piel, disminución de la tasa de sudoración, disminución de la pérdida de calor, aumento de la temperatura central, produciendo deterioro del rendimiento deportivo o incluso de la salud del individuo.

Mucho se ha investigado con relación a los efectos de la deshidratación en modalidades deportivas de esfuerzos prolongados, donde sus resultados están bien documentados (Montain, Smith, Mattot, Zientara, Jolesz y Sawka, 1998) y están de acuerdo en que provoca un impacto progresivamente negativo sobre el rendimiento en ejercicio, aún a niveles bajos de deshidratación entre 1 y $2 \%$ del peso corporal (Maughan, 2003). Los principales efectos del estado de hipohidratación sobre el rendimiento son: disminución del $\mathrm{VO}_{2}$ máximo, disminución del tiempo del ejercicio hasta la fatiga a intensidades submáximas (González-Alonso y Coyle, 1998; Nybo, Nielsen y González-Alonso, 2001), disminución de las reservas de glucógeno hepático y aumento de la frecuencia cardiaca (Aragón-Vargas et al, 1999 y Murray, 1996).

Mientras tanto, los efectos de la deshidratación en actividades de corta duración no son tan claros.

\section{La deshidratación y el rendimiento anaeróbico:}

Las investigaciones que han examinado el rendimiento en actividades anaeróbicas y el metabolismo presentan resultados un poco confusos. Jacobs (1980), que aplicó a los sujetos la prueba de rendimiento anaeróbico en cicloergómetro (Wingate test) bajo estado de euhidratación y con deshidrataciones del $2 \%, 4 \%$ y $5 \%$ de su peso corporal total. El autor encontró que la deshidratación no afectó los resultados de las pruebas de rendimiento anaeróbico o en los niveles de lactato post ejercicio. Sin embargo, Webster,
Rutt y Weltman (1990) usando un protocolo similar de rendimiento anaeróbico observaran que la deshidratación de $4.9 \%$ del peso corporal desminuyó el rendimiento anaeróbico y el nivel de lactato pico de los sujetos. Aunque ambas investigaciones usaran pruebas similares de rendimiento anaeróbico sus resultados fueron totalmente diferentes.

Esas diferencias en los resultados podrían ser explicados por la diferencia en los métodos para provocar la deshidratación en los sujetos. Jacobs (1980) utilizó un método agudo pasivo (sauna) para lograr que los sujetos se deshidratasen, mientras que Webster et al (1990) deshidrataran los sujetos por medio de privación de agua, ejercicio y utilizando ropas pesadas durante un período de 12 horas.

Utilizando una prueba submáxima de carrera con duración de un minuto en la banda sin fin, Houston, Martin, Green y Thomson (1981) reportan que la deshidratación de $8 \%$ del peso corporal no afecta el resultado en esta prueba de rendimiento anaeróbico. Mientras tanto, en el mismo año (Nielsen, Kubica, Bonnesen y Rassmussen, 1981) se publicó otra investigación donde los sujetos realizaran una prueba submáxima en cicloergómetro en estados de euhidratación e hipohidratación de $3 \%$ del peso corporal. Los autores observaron que el rendimiento anaeróbico disminuyó cuando los sujetos fueron deshidratados por diuréticos $(-18 \%)$, sauna $(-35 \%)$ y por ejercicio (-44\%).

Las investigaciones modernas sobre deshidratación usan dos distintos modelos experimentales básicos para lograr la deshidratación (Murray y Udermann 2003):

1. Pasivo: Pérdidas de fluidos inducidas por diuréticos, sauna y restricción de fluidos antes del ejercicio.

2. Activo: Pérdidas de fluidos inducidas por ejercicio resultado de una deshidratación progresiva.

Entretanto, debido a la existencia de investigaciones que utilizan combinaciones 
de los métodos pasivos y activos como medio de provocar la deshidratación, se hizo necesario concentrar esas investigaciones en un tercer grupo, que se le denominó como "Mixto". En este grupo fueran incluidas principalmente las investigaciones donde participaron atletas provenientes de deportes de lucha (judo, boxeo y wrestling), quienes por competir en distintas categorías de peso, en muchos casos, utilizan la deshidratación como forma de perder peso y competir en categorías de peso más bajas. En la mayoría de los casos, en días previos a la competición, los luchadores combinan la práctica de ejercicio en ambiente caliente y los baños sauna (Webster, Rutt y Weltman, 1988); ejercicio utilizando ropas pesadas con restricción de fluidos (Webster, Rutt y Weltman, 1990) para alcanzar el peso deseado.

\section{La deshidratación "pasiva"}

La mayor parte de las investigaciones analizadas que utilizaran el sauna como medio de deshidratación, reportan no haber encontrado diferencia significativa en los resultados de las pruebas de rendimiento anaeróbico entre las condiciones euhidratado y deshidratado. Como se puede observar en la Tabla 1., tampoco se encontró una relación entre los porcentuales de deshidratación y los cambios en el rendimiento.

Mientras tanto, los estudios encontrados que utilizaron diuréticos para inducir la deshidratación, reportaron disminución de $21 \%$ en la carga máxima de trabajo con un porcentual de deshidratación de 4,1\% del peso corporal (Caldwell, Ahonen y Nousiainen, 1984) y menos $18 \%$ en la capacidad anaeróbica submáxima con 3\% de deshidratación (Nielsen et al, 1981).

\section{La deshidratación "Activa"}

Este método de deshidratación está dividido en dos grupos:
1.Deshidratación provocada por ejercicio (Tabla 2.), donde todos los resultados reportados por los investigadores demuestran efectos negativos de la deshidratación sobre: rendimiento en judo utilizando la prueba "Special Judo Fitness Test" (Da Silveira, 2005), carga máxima de trabajo (Caldwell et al, 1984) y en la capacidad anaeróbica submáxima (Nielsen et al, 1981) en prueba de cicloergómetro.

2.Deshidratación provocada por ejercicio en el calor (Tabla 2.), en este grupo están las investigaciones donde el proceso de deshidratación ocurrió en laboratorios de clima controlado. Yoshida, Takanishi, Nakai, Yorimoto y Morimoto, (2002) reportaron una disminución de $4 \%$ en la potencia anaeróbica máxima donde los sujetos fueron deshidratados a un nivel de $3.1 \%$ del peso corporal. Mientras tanto, Nybo et al (2001) reportaron un aumento de la frecuencia cardiaca submáxima con deshidratación de $4 \%$, pero no encontraron diferencias estadísticamente significativas en las variables: frecuencia cardiaca máxima y concentración de glucógeno muscular.

\section{El método "mixto" de deshidratación}

El método de deshidratación mixta se caracteriza por la combinación de los métodos activo y pasivo, que tienen como objetivo alcanzar niveles relativamente altos de hipohidratación en períodos superiores a 24 horas (Webster et al, 1990).

Los resultados de lo estudios de Webster et al (1988) y Webster et al (1990), en los cuales los sujetos participantes eran luchadores, están de acuerdo en que la pérdida de $5 \%$ del peso corporal en un período de $36 \mathrm{~h}$, por medio de práctica combinada de ejercicio en ambiente caliente y el baño sauna, así como los ejercicios utilizando ropas pesadas y restricción de fluidos, causan deterioro importante en el rendimiento de las variables relacionadas con el metabolismo anaeróbico, como se puede apreciar en la Tabla 3. 
Tabla 1. Efectos del método pasivo de deshidratación utilizando sauna o diurético.

\begin{tabular}{lccll}
\hline Método Pasivo & $\mathbf{N}$ & $\boldsymbol{\Delta} \mathbf{P C} \%)$ & \multicolumn{1}{c}{ Resultado } & \multicolumn{1}{c}{ Referencia } \\
\hline \multirow{2}{*}{ Sauna } & 10 & -1.5 & $\downarrow 5.6 \%$ F. Máx. (press de banca) & Schoffstall (2001) \\
& 10 & -3.8 & $\downarrow 7.8 \%$ F. Isom. de piernas (voleibolistas) & Vitasalo et al (1987) \\
& 7 & -4 & NS Tiempo hasta la fatiga (exten. de rodillas) & Greiwe et al (1998) \\
& 7 & -4 & NS Numero max. de repet. (flex. de codos) & Greiwe et al (1998) \\
& 16 & -4.1 & NS Glucógeno muscular & Caldwell et al (1984) \\
& 16 & -4.1 & $\downarrow 23$ W Carga máx. de trabajo (cicloregómetro) & Caldwell et al (1984) \\
& 16 & -4.1 & $\downarrow$ Significativamente Lactato en la sangre & Caldwell et al (1984) \\
& 6 & -3 & $\downarrow 35 \%$ Capacidad anaeróbica submáxima & Nielsen et al (1981) \\
& 5 & -2 & NS Rendimiento anaeróbico (Wingatte) & Jacobs (1980) \\
& 5 & -3 & NS Rendimiento anaeróbico (Wingatte) & Jacobs (1980) \\
& 5 & -5 & NS Rendimiento anaeróbico (Wingatte) & Jacobs (1980) \\
& 16 & -4.1 & $\downarrow 21 \%$ Carga máx. de trabajo & Caldwell et al (1984) \\
& 6 & -3 & $\downarrow 18 \%$ Capacidad anaeróbica Submáxima & Nielsen et al (1981) \\
\hline
\end{tabular}

$\mathrm{N}$ (cantidad de sujetos); $\triangle \mathrm{PC}$ (\% de peso corporal perdido por deshidratación); NS (No hubo diferencias significativa).

Tabla 2. Efectos del método activo de deshidratación a través de ejercicio o ejercicio en el calor.

\begin{tabular}{|c|c|c|c|c|}
\hline Método Activo & $\mathbf{N}$ & $\Delta \mathrm{PC}(\%)$ & Resultado & Referencia \\
\hline \multirow[t]{3}{*}{ Ejercicio } & 16 & -1.88 & $\downarrow 7 \%$ Prueba de rendimiento (judo) & Da Silveira (2005) \\
\hline & 16 & -2 & $\begin{array}{l}\downarrow 7 \mathrm{~W} \text { Carga máx. de trabajo } \\
\text { (cicloergómetro) }\end{array}$ & Caldwell et al \\
\hline & 6 & -3 & $\downarrow 44 \%$ Capacidad anaeróbica Submáxima & Nielsen et al (1981) \\
\hline \multirow[t]{4}{*}{ Ejercicio+Calor } & 6 & -4 & $\begin{array}{l}\uparrow \text { FC Submáx. en } 8 \text { lat } / \text { min. } \\
\text { (cicloergómetro) }\end{array}$ & Nybo et al (2001) \\
\hline & 6 & -4 & NS FC Máx. (cicloergómetro) & Nybo et al (2001) \\
\hline & 6 & -4 & NS Glucógeno muscular & Nybo et al (2001) \\
\hline & 18 & -3.1 & $\downarrow 4 \%$ Potencia anaeróbica máxima & $\begin{array}{l}\text { Yoshida et al } \\
(2002)\end{array}$ \\
\hline
\end{tabular}

$\mathrm{N}$ (cantidad de sujetos); $\triangle \mathrm{PC}$ (\% de peso corporal perdido por deshidratación); NS (No hubo diferencias significativa). FC (Frecuencia Cardiaca Máxima. 
Tabla 3. Efectos del método mixto de deshidratación a través de ejercicio, calor y sauna o ejercicio utilizando ropa pesada y restricción de fluidos.

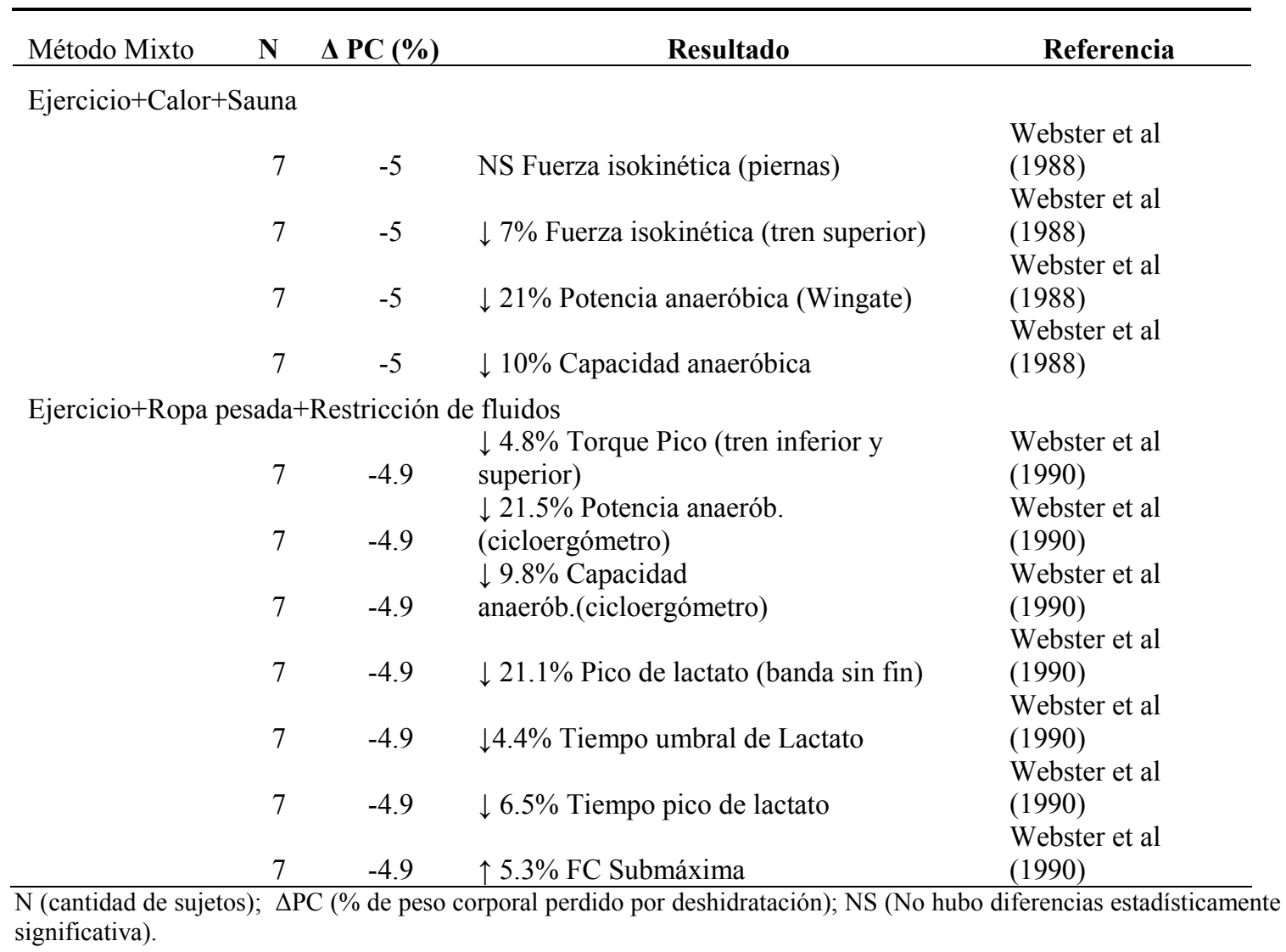

\section{DISCUSIÓN}

En cuanto a los mecanismos relacionados con la disminución del rendimiento anaeróbico inducido por la deshidratación, Sawka y Pandolf (1990) y Yoshida et al (2002) especulan que pueden estar relacionados con dos factores principales:

1. La reducción del contenido de agua en el músculo es probablemente seguido por una concentración electrolítica alterada, la cual puede perturbar el potencial electroquímico de la membrana celular de tal modo que afecta la potencia anaeróbica.
2. el aumento de la activación simpática debido a la deshidratación puede estar relacionado con una mayor utilización del glucógeno muscular.

Los efectos de la deshidratación en el rendimiento anaeróbico no parecen claros. Entretanto, así como lo que ocurre en el rendimiento aeróbico, las diferencias en los resultados del rendimiento anaeróbico parecen estar relacionados con algunos factores como:

1. La magnitud de cambio en el peso corporal, donde cuanto mayor el porcentaje de deshidratación mayores son los efectos negativos; 
2. El tiempo en que los sujetos estuvieran bajo proceso de deshidratación, en el cual cuanto mayor es el período de tiempo en que los sujetos estuvieron deshidratándose mayores fueron las pérdidas;

3. Los métodos de deshidratación y principalmente si hubo combinación de estos métodos. Porque según las investigaciones revisadas el método mixto presenta como característica un alto porcentaje de deshidratación alcanzados en largos períodos, lo que hace que los sujetos permanezcan ejercitándose bajo un estado de hipohidratación, que resulta en pérdidas aún mayores de electrolitos, disminución de las reservas musculares y hepáticas de glucógeno que conlleva al deterioro del rendimiento anaeróbico.

Para que se tenga resultados más conclusivos al respecto de los efectos de la deshidratación en el rendimiento y metabolismo anaeróbico, se hace necesario más investigaciones en ésta área, principalmente referentes a los mecanismos fisiológicos implicados.

\section{RECOMENDACIONES:}

$$
\begin{aligned}
& \text { El Colegio Americano de } \begin{array}{r}
\text { Medicina } \\
\text { Deportiva (1996), tras muchas }
\end{array}
\end{aligned}
$$
investigaciones, emitió su posición acerca del protocolo óptimo de hidratación que se debe seguir al hacer ejercicio de larga duración o más de una sesión de ejercicios diarios. En resumen aconseja que:

1. Se debe tener una dieta balanceada y una ingesta de líquido adecuada todo el día.

2. Se debe tomar $500 \mathrm{ml}$ de líquido dos horas antes de iniciar la actividad física.

3. Durante el ejercicio se debe ingerir la máxima cantidad tolerable a intervalos regulares, lo que podría ser cantidades entre 125 y $300 \mathrm{ml}$ cada 15 o 20 minutos.

4. El líquido debe estar a $15^{\circ} \mathrm{C}$, y poseer un sabor agradable.
5. Si el evento tarda más de una hora, el líquido debe tener entre $4 \%$ y $8 \%$ de carbohidratos, además de entre 0.5 y 0.7 gr de sodio por litro de agua.

6. Inmediatamente después del evento, se debe seguir ingiriendo líquido hasta reponer todo el que se perdió.

\section{REFERENCIAS}

American Collage of Sport Medicine (1996). ACSM position stand on exercise and fluid replacement. Medicine and Science Sport Exercise, 28(1), ivii.

Aragón-Vargas L. F., Maughan, R. J., Rivera-Brow, A., Meyer, F., Murray, R., de Barros, T. L., García, P. L., Sarmiento, J. M., Arroyo, F., Javornik, R., Matsudo, V. K. R., Salazar, W. y Lentini, M. (1999). VII Simposio Internacional de Actualización en Ciencias del Deporte. Rosario, Argentina, Editorial Biosystem, servicio educativo. 222-230.

Caldwell, J. E., Ahonen, E., y Nousiainen, U. (1984). Differential effects of sauna -diuretic - and exercise - inducid hypohydration. Journal of Apply.Physiology, 57(4), 1018-1023.

Cheung, S. S., Mclellan, T. M. y Tenaglia, S., (2000). The thermophysiology of uncompensable heat stress: physiological manipulation and individuals characteristics. Sports Med, 29(5), 239-259.

Da Silveira, U. F. (2005). El efecto de la deshidratación en el rendimiento de atletas de judo. En Memorias del III Congreso Centroamericano de Educación Física, Deporte y Recreación. Ciudad de Panamá, Panamá: Universidad de Panamá - Instituto Panamericano de Educación Física, 1 (44).

Franchini, E. (1a. ed). (2001). Judo: Desempenho Competitivo. São Paulo: Brasil.

González-Alonso, J., y Coyle, E. F. (1998). Efectos fisiológicos de la deshidratación. ¿Por qué los deportistas deben ingerir líquidos durante el ejercicio en el calor? Apunts-Educación Física y Deportes, 54(4), 46-52.

Greiwe, J. S., Staffey, K. S., Melrose, D. R., Narve, M. D. y Knowlton, R. G. (1998). Effects of dehydration on isometric muscular strength and endurance. Med. Sci. Sports Sci., 30(2), 284-288.

Houston, M. E., Martin, D. A., Green, H. J. y Thomson, J. A. (1981). The effect of rapid weight loss on physiological functions in wrestlers. Phys. Sportsmed, 9,73-78.

Horswill, C. A. (1998). Effective fluid replacement. Int $J$ Sport Nut, 8, 175-195. 
Koeppen, B. M., y Stanton, B. A. (2001). Renal Physiology. ( $3^{\text {a }}$.ed., pp. 49-73). United Sates of America: Mosby Imcorporation.

Jacobs, I. (1980). The effects of thermal dehydration on performance of the Wingate anaerobic test. Int. J. Sports Med., 1, 21-24.

Lamb, D. R., y Shehata, A. H. (1999). Beneficios y limitaciones de la pre-hidratación. Sport Science Exchange, 24.

Lieberman, H. R., Bathalon, G. P., Falco, C. M., Kramer, F. M., Morgan III, C. A., y Niro, P. (2004). Severe decrements in cognition function and mood induced by sleep loss, heat, dehydration and undernutrition during simulated combat. Biol Phychiatry, 57, 422429.

Maughan, R. J. (1998). Restoration of water and electrolyte balance after exercise. Int J Sports Med, 19, 136-138.

Maughan, R. J. (2003). Impact of mild dehydration on wellness and on exercise performance. European Journal of Clinical Nutrition, 57(2), 19-23.

McArdle, W., Katch, F., y Katch, V. (1986). Fisiología del ejercicio: Energía nutrición y rendimiento humano ( ${ }^{\mathrm{a}}$ ed.). Madrid: Alianza Editorial

Murray, S. R. (1996). Dehydration, hyperthermia, and athletes: Science and practice. Journal of Athletic Training, 31(3), 248-252.

Murray, S. R. y Udermann, B. E. (2003). Fluid replacement: A historical perspective and critical review. International Sports Journal, 7(2), 58-73.

Montain, S. J., Smith, S. A., Mattot, R. P., Zientara, G. P., Jolesz, F. A. y Sawka, N. (1998). Hypohydration effects on skeletal muscle performance and metabolism: a 31P-MRS study. J. Appl. Physiol. 84(6), 1889-1894.

Nielsen, B., Kubica, R., Bonnesen, A., Rassmussen, I. B., Stoklosa J. y Wilk, B.(1981). physical work capacity after dehydration and hyperthermia: a comparison of the effect of exercise versus passive heating and sauna and diuretic dehydration. Scand. J. Sport Sci. 3, 210.

Nybo, L., Jensen, T., Nielsen, B. y González-Alonso, J. (2001). Effects of marked hyperthermia with and without dehydration on Vo2 kinetics during intense exercise. J Appl Physiol, 90, 1057-1064.

Ruiz, J. R., Mesa, J. L., Pérez, F. M., Sáinz, A. G. y Garzón, M. J. (2003). Hidratación y rendimiento: pautas para una elusión efectiva de la deshidratación por ejercicio. Apunts. Educación Física y Deportes, 70, 26-33.

Sawka, M. N. y Pandolf, K. B. (1990). Effects of body water loss son physiological function and exercise performance. Perspectives in exercise science and sports medicine: Fluid Homeostasis During Exercise ( $3^{\mathrm{a}}$ ed.) C.V.
Gisolfi y D.R. Lamb. Indianapolis: Benchmark.

Schoffstall, J. E., Branch,J. D., Leutholtz, C. y Swain, D. P. (2001). Effects of dehydration and rehydration on the one-repetition maximum bench press of weight-trained males. $J$ Strength and Conditioning Research, 15(1), 102-108.

Shirreffs, S. M. (2000). Markers of hydration status. $J$ Sports Med Phys Fitness, 40, 80-84

Shirreffs, S. M. y Maughan, R. J. (2001). Rehydration and recovery of fluid balance. En: Maughan, R., y Murray, R. Sports Drinks: Basic science and practical aspects (pp 29-43). United States of America: CRC Press LLC.

Solera-Herrera, A. (2003). Efectos de la deshidratación y la rehidratación en la efectividad del tiro libre de baloncesto. Revista de Ciencias del Ejercicio y la Salud, 3 (1), 35-42.

Vitasalo, J. T., Kyrolainen, H., Bosco, C. y Altan, M. B. (1987). Effects of rapid weight reduction on force production and velocity jumping height. Int. J. Sports Med. 8, 281-285.

Wilmore, H. J. y Costill, D. L. (1999). Fisiología del esfuerzo y del deporte (2a ed.). Barcelona: Paidotibo.

Webster, S., Rutt, R. y Weltaman, A. (1988). Effects of typical dehydration practices on performance. Med. Sci. Sports Exerc., 20.

Webster, S., Rutt, R. y Weltaman, A. (1990). Physiolocal effects of a weight loss regimen practiced by college wrestlers. Med. Sci. Sports Exerc., 22(2), 229-234.

Yoshida, T., Takanishi, T., Makai, S., Yorimoto, A. y Morimoto, T. (2002). The critical level of water deficit causing a decrease in human exercise performance: a practical field study. Eur J Appl Physio, 87, 529-534. 